\title{
Neutron Noise Patterns from Coupled Fuel-Assembly Vibrations
}

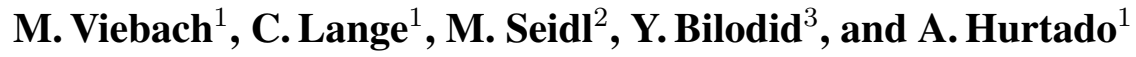 \\ ${ }^{1}$ Technische Universität Dresden, Chair of Hydrogen and Nuclear Energy \\ George-Bähr-Straße 3b, D-01069 Dresden, Germany \\ ${ }^{2}$ PreussenElektra GmbH, Tresckowstrae 5, D-30457 Hannover, Germany \\ ${ }^{3}$ Helmholtz-Zentrum Dresden-Rossendorf, Reactor Safety Division \\ Bautzner Landstraße 400, D-01328 Dresden, Germany
}

marco.viebach@tu-dresden.de, marcus.seidl@preussenelektra.de, y.bilodid@hzdr.de

\begin{abstract}
The neutron flux fluctuation magnitude of KWU-built PWRs shows a hitherto unexplained correlation with the types of loaded fuel assemblies. Also, certain measured long-range neutron flux fluctuation patterns in neighboring core quadrants still lack a closed understanding of their origin. The explanation of these phenomena has recently revived a new interest in neutron noise research.

The contribution at hand investigates the idea that a synchronized coolant-driven vibration of major parts of the fuel-assembly ensemble leads to these phenomena. Starting with an assumed mode of such collective vibration, the resulting effects on the time-dependent neutron-flux distribution are analyzed via a $D Y N 3 D$ simulation. A three-dimensional representation of the time-dependent bow of all fuel assemblies is taken into account as a nodal $D Y N 3 D$ feedback parameter by time-dependent variations of the fuel-assembly pitch. The impact of its variation on the cross sections is quantified using a cross-section library that is generated from the output of corresponding CASMO5 calculations.

The $D Y N 3 D$ simulation qualitatively reproduces the measured neutron-flux fluctuation patterns. The magnitude of the fluctuations and its radial dependence are comparable to the measured details. The results imply that collective fuel-assembly vibrations are a promising candidate for being the key to understand long-known fluctuation patterns in KWU built PWRs. Further research should elaborate on possible excitation mechanisms of the assumed vibration modes.
\end{abstract}

KEYWORDS: Neutron noise, KWU, PWR

\section{INTRODUCTION}

During the previous two decades, the neutron flux fluctuations (also referred to as neutron noise) of PWRs built by KWU (Kraftwerk Union AG) have exhibited unexplained changes of their magnitude (cf. Ref. [1]). The phenomenon has attracted new attention to neutron flux fluctuations and has called for corresponding research efforts aiming on understanding it (see e. g. Refs. [2-6]). 
The analysis of measured fluctuation data has drawn attention to long-known but also unexplained long-range correlations (see e.g. Ref. [5]): in-phase behavior for axially aligned detectors and for detectors in the same core quadrant and out-of-phase behavior for detectors in opposite core regions. The fluctuation magnitudes quantified by the normalized standard deviation are in the range of percents in Vorkonvoi PWRs (cf. Refs. [4-6]). They have higher values in the outer fuelassembly rings and lower values in more central regions. The axial shape is C-like (cf. Refs. $[4,6])$.

The change in magnitude is correlated with the number of fuel assemblies of a certain type. Therefore, some recent activities consider that fuel assembly vibrations trigger the phenomena described above including the change in magnitude. Reference [5] investigates the so-called "reflector effect", which means a correlated fluctuation of the water-layer thickness between the outer fuel assemblies and the core shroud resulting from a synchronous vibration of all fuel assemblies. Corresponding simulations with the system code $D Y N 3 D$ [7] yielded neutron flux fluctuation correlations similar to those of the measurements. On the other hand, the induced fluctuation magnitudes were too small by about one order magnitude and their radial dependence was too strong. These two shortcomings let to the suggestion of a perturbation source located in the outer fuel-assembly rings rather than in the reflector.

According to Ref. [8], the outer fuel-assembly rings may permanently touch the core shroud and/or one another during operation as a result of coolant-flow forces, irradiation-induced creep, and thermal expansion. As proposed in Ref. [5], the corresponding lateral fixation of the outer fuel assemblies in conjunction with a synchronized vibration of the non-fixed fuel assemblies could make a perturbation source of required magnitude in the suggested region. Because this scenario corresponds to fluctuating distances between outer and inner fuel assemblies, it is referred to as "fuel-assembly-pitch effect". The paper at hand, studies this idea via a new DYN3D simulation.

\section{SIMULATION OF NEUTRON FLUCTUATION PATTERNS FROM COUPLED FUEL-ASSEMBLY VIBRATIONS}

\subsection{Description of the model}

The model aims on investigating the impact of a synchronized fuel-assembly vibration on the neutron-flux fluctuation patterns. It consists of a simplified model of the fuel-assembly vibration and of a $D Y N 3 D$ model representing a four-loop KWU PWR. Both models are coupled via the interaction cross sections.

\subsubsection{Neutron kinetics and thermal hydraulics}

Figure 1 presents the nodal setup of the employed PWR model used for the DYN3D simulation. It represents a KWU Vorkonvoi PWR at $100 \%$ power at end of cycle. The model is described in more detail in Ref. [5]. The used DYN3D version solves the time-dependent three-dimensional neutron transport equation considering two energy groups and using the diffusion approximation for the angular dependency and nodal methods for the spatial dependency. The equations are coupled to the intrinsic one-dimensional thermal-hydraulics module, which represents the coolant flow by four equations for each individual coolant channel, and to the one-dimensional heat-conduction equation for the fuel rods. Reference [7] may be consulted for more details. 


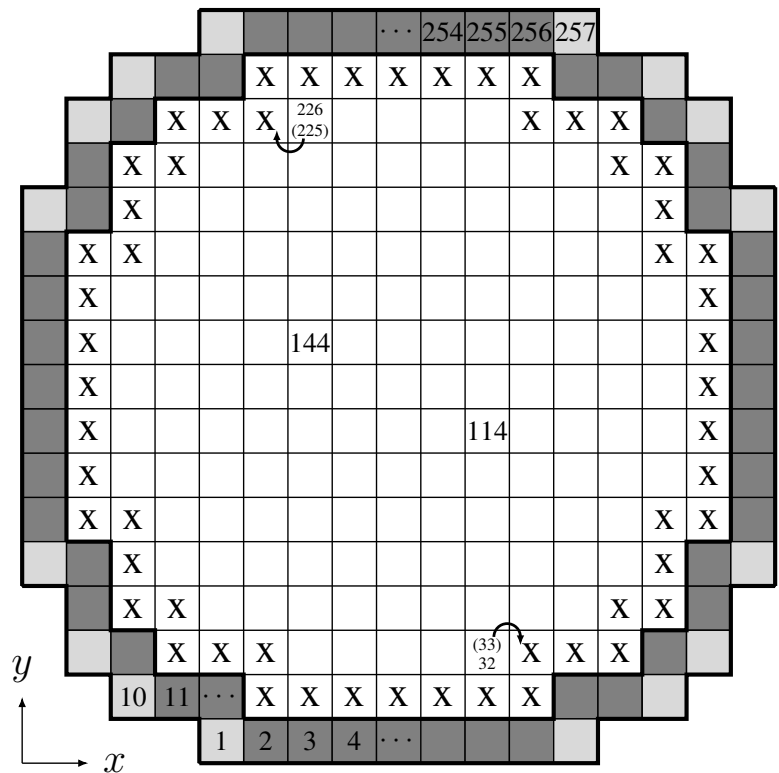

(a) Radial-azimuthal.

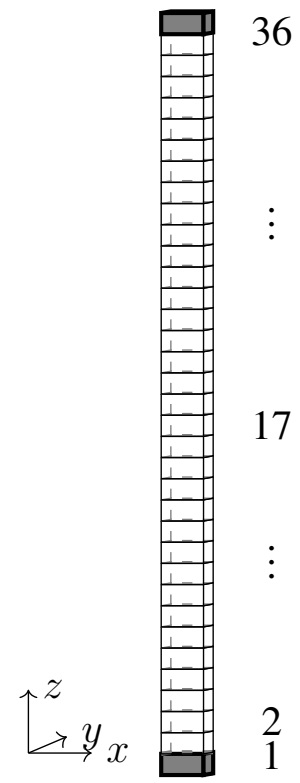

(b) Axial.

Figure 1: Spatial setup of the reactor in $D Y N 3 D$. The fuel-assembly regions (white areas) and the the reflector regions (gray areas) are given by respective homogenized material parameters. Crosses indicate laterally fixed fuel assemblies for the mechanical model.

For the simulation of neutron flux fluctuations, the system is initialized by a steady-state calculation. In the subsequent time-dependent calculation, the system is externally perturbed by a variation of the fuel-assembly-pitch variable $p_{i}(z, t), i=1,2, \ldots, 193, t>0 \mathrm{~s}$. This variable was added to the $D Y N 3 D$ feedback parameters to incorporate time-dependent fuel-assembly bow, i. e. variations of the fuel-assembly distances $d_{i j}(z, t), j=1,2, \ldots, 8$ in an approximate manner:

$$
p_{i}(z, t):=\frac{1}{4} \cdot \sum_{j_{i} \in\{1,2,7,8\}} d_{i j_{i}}(z, t) .
$$

The fuel assemblies indexed $j_{i} \in\{1,2\}$ are the $\mathrm{x}$-direction neighbors of the $i$ th fuel assembly and those indexed $j_{i} \in\{7,8\}$ are its y-direction neighbors (see Fig. 2 in Sec. 2.1.2 for the indexing). In order to provide the corresponding branch for the variable $p_{i}(z, t)$ in the $D Y N 3 D$ cross-section library, which covers the full set of group constants (also assembly discontinuity factors, e. g.), a series of CASMO5 [9] lattice-code runs were performed under variation of the fuel-assembly pitch $p .{ }^{*}$ The time series $p_{i}(z, t)$ are provided from the mechanical model (Sec. 2.1.2) and are loaded from an external file during the $D Y N 3 D$ run. After the $D Y N 3 D$ simulation, the fluctuations of the nodal power density are evaluated as a measure for the neutron flux fluctuations.

${ }^{*}$ The $D Y N 3 D$ cross-section library was generated based on the two sets of group constants corresponding to $p=p_{\text {nominal }} \pm 1.6 \mathrm{~mm}$, only. Because the impact of $p$ was found to be quasi linear, applicability of linear interpolation between the two extreme values is assumed. The calculation of the group constants at respective sampling points of the other branches $\left(T_{\text {fuel }}, T_{\text {moderator }}, \rho_{\text {moderator }}\right)$ relevant for the simulation of neutron flux fluctuations was internally performed by CASMO5. 


\subsubsection{Mechanical model}

For both directions $d=\mathrm{x}, \mathrm{y}$, each fuel assembly is represented by an independent homogeneous bar. Both the deflection $w_{i, \mathrm{x}}$ in $\mathrm{x}$-direction and the deflection $w_{i, \mathrm{y}}$ in y-direction are approximated by a cosine bow-shape function and a driven and damped harmonic oscillator having amplitude $A_{i, \mathrm{x}}(t)$ and $A_{i, \mathrm{y}}(t)$, resp.:

$$
\begin{aligned}
w_{i, d} & =\cos \left(\frac{\pi}{L} z\right) \cdot A_{i, d}(t), z \in\left[-\frac{L}{2}, \frac{L}{2}\right], d=\mathrm{x}, \mathrm{y} \\
\frac{F_{i, d}}{m_{i}}(t) & =\frac{\mathrm{d}^{2}}{\mathrm{~d} t^{2}} A_{i, d}(t)+2 \delta_{i} \omega_{0, i} \frac{\mathrm{d}}{\mathrm{d} t} A_{i, d}(t)+\omega_{0, i}^{2} A_{i, d}(t), t>0 \mathrm{~s}, d=\mathrm{x}, \mathrm{y}
\end{aligned}
$$

with the length $L$ of the beam, the effective mass $m_{i}$, the natural frequency $\omega_{0, i}$ of the undamped system, and the damping coefficient $\delta_{i}$. The driving forces $F_{i, d}$ are composed of an "external" contribution $F_{i, d \text {,ext }}$ and an internal contribution $F_{i, d \text {,int }}$

$$
F_{i, d}(t)=F_{i, d, \mathrm{ext}}(t)+F_{i, d, \text { int }}(t)
$$

The "external" contribution representing the fuel-assembly drive by coolant flow fluctuations is modeled by Gaussian random numbers having zero mean $\mu\left(F_{i, d \text {,ext }}\right)$ and non-zero standard deviation $\sigma\left(F_{i, d \text {,ext }}\right)$. They are sampled with the rate $\delta t\left(F_{i, d \text {,ext }}\right)$. The internal contribution represents the drive by the adjacent fuel assemblies modeled as

$$
F_{i, d, \text { int }}(t)=\sum_{j_{i}=1}^{8} k_{i j_{i}, d}\left(A_{i, d}(t)-A_{j_{i}, d}(t)\right)
$$

with the amplitude $A_{j_{i}}$ of the $j_{i}$ th adjacent fuel assembly $\left(j_{i}=1,2, \ldots, 8\right)$, and the coupling constants $k_{i j_{i}, d}$. The coupling constants are non-uniform. The situation is illustrated in Fig. 2. In each direction, $d=\mathrm{x}, \mathrm{y}$, eight forces are acting on the central fuel assembly. In x-direction and $\mathrm{y}$-direction, the eight respective summands on the right-hand side of Eq. (5) can be identified with

$$
\begin{aligned}
& F_{i, \mathrm{x}, \text { int }}(t)=F_{\mathrm{f}, i 1}+F_{\mathrm{f}, i 2}+F_{\mathrm{s}, i 7}+F_{\mathrm{s}, i 8}+\sum_{j_{i}=3}^{6} F_{\mathrm{r}, i j_{i}, \mathrm{x}}, \\
& F_{i, \mathrm{y}, \text { int }}(t)=F_{\mathrm{f}, i 7}+F_{\mathrm{f}, i 8}+F_{\mathrm{s}, i 1}+F_{\mathrm{s}, i 2}+\sum_{j_{i}=3}^{6} F_{\mathrm{r}, i j_{i}, \mathrm{y}}
\end{aligned}
$$

with $F_{\mathrm{f}, i 1}, F_{\mathrm{f}, i 2}, F_{\mathrm{f}, i 7}$, and $F_{\mathrm{f}, i 8}$ representing face-to-face forces, $F_{\mathrm{s}, i 7}, F_{\mathrm{s}, i 8}, F_{\mathrm{s}, i 1}$, and $F_{\mathrm{s}, i 2}$ representing shear forces within one fuel-assembly row, and $F_{\mathrm{r}, i j_{i}, \mathrm{x}}$ and $F_{\mathrm{r}, i j_{i}, \mathrm{y}}\left(j_{i}=3,4,5,6\right)$ representing shear forces between diagonally adjacent fuel assemblies. 


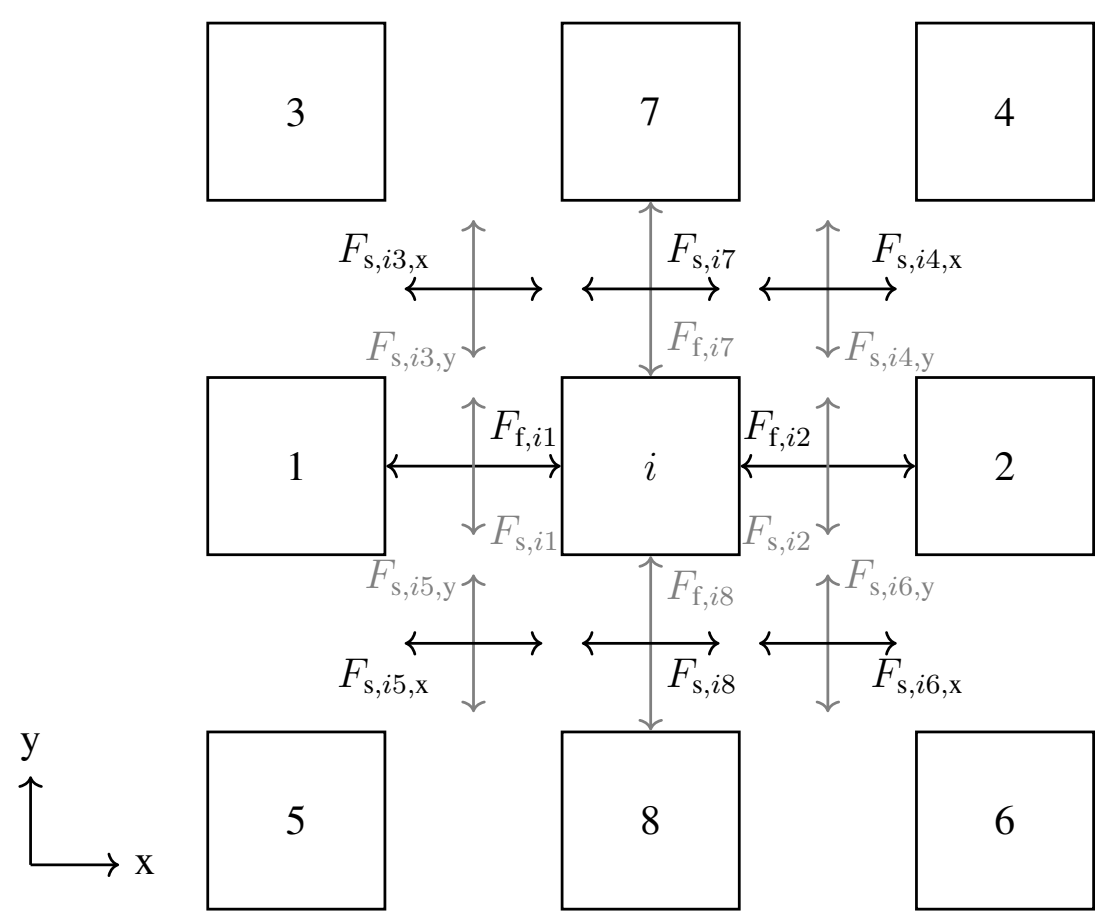

Figure 2: Coupling forces of the $i$ th fuel assembly with its neighbors $j_{i}=1,2, \ldots, 8$.

In the simulations, equal coupling constants $k_{\mathrm{f}}, k_{\mathrm{s}}$, and $k_{\mathrm{r}}$ are assumed, i.e.

$$
\begin{gathered}
k_{\mathrm{f}}=k_{i j_{i}, d}: F_{\mathrm{f}, i j_{i}}=k_{\mathrm{f}}\left(A_{i, d}(t)-A_{j_{i}, d}(t)\right), i=1,2, \ldots, 193,\left(j_{i}, d\right)=(1, \mathrm{x}),(2, \mathrm{x}),(7 \mathrm{a}) \\
\\
\begin{aligned}
&(7, \mathrm{y}),(8, \mathrm{y}), \\
& k_{\mathrm{s}}=k_{i j_{i}, d}: F_{\mathrm{s}, i j_{i}}=k_{\mathrm{s}}\left(A_{i, d}(t)-A_{j_{i}, d}(t)\right), i=1,2, \ldots, 193,\left(j_{i}, d\right)=(1, \mathrm{x}),(2, \mathrm{x}),(7 \mathrm{~b}) \\
&(7, \mathrm{y}),(8, \mathrm{y}), \\
& k_{\mathrm{r}}=k_{i j_{i}, d}: F_{\mathrm{r}, i j_{i}, d}=k_{\mathrm{d}}\left(A_{i, d}(t)-A_{j_{i}, d}(t)\right), i=1,2, \ldots, 193, j_{i}=3,4,5,6, \quad(7 \mathrm{c}) \\
& d=\mathrm{x}, \mathrm{y} .
\end{aligned}
\end{gathered}
$$

The outer fuel-assembly ring is assumed to be laterally fixed (see crossed channels in Fig. 1a). In the model, this is maintained by setting the coupling constants $k_{\mathrm{f}}, k_{\mathrm{s}}, k_{\mathrm{r}}$ and the "external" driving forces $F_{i, d, \text { ext }}$ of the fixed fuel assemblies to zero.

Table 1 gives the parameter values used for the mechanical simulation, which is performed in $M A T L A B$ [10] using ode45() for integrating the set of ordinary differential equations. The two directions $d=\mathrm{x}, \mathrm{y}$ are treated independently and prior to the $D Y N 3 D$ run. From the resulting amplitudes $A_{i, d}$, the fuel-assembly distances $d_{i j_{i}}(t)$ follow as

$$
d_{i j_{i}}(z, t)=\left(A_{i, d}(t)-A_{j_{i}, d}(t)\right) \cdot \cos \left(\frac{\pi}{L} z\right),\left(j_{i}, d\right)=(1, \mathrm{x}),(2, \mathrm{x}),(7, \mathrm{y}),(8, \mathrm{y})
$$

and the fuel-assembly-pitch variable $p_{i}(z, t)$ follows from Eq. (1). In Eqs. (1) and (8), the axial shape function can be factored out such that at time $t, 193$ axially independent pitch values $p_{i}(t)=$ $p_{i}(z, t) / \cos \left(\frac{\pi}{L} z\right)$ are provided to $D Y N 3 D$. Multiplication by $\cos \left(\frac{\pi}{L} z\right)$ is performed in $D Y N 3 D$. 
It has to be emphasized that the chosen values of the coupling constants represent a configuration that yields a quasi-synchronous vibration of all non-fixed fuel assemblies, although all of them are driven independently. The driving force is set to a value which provides a maximum pitch variation $\lesssim \pm 1.6 \mathrm{~mm}$. Furthermore, the mechanical model is a strong simplification of the actual conditions. These are accepted here because the purpose of this paper is the demonstration of the impact of a synchronized vibration of all fuel assemblies but the outermost ring. Future studies will investigate how a synchronous vibration of the fuel assemblies could actually be triggered (including contact, large-dimension coolant fluctuations, or core-barrel vibrations).

Table 1: Parameters used for the mechanical model $\left(i=1,2, \ldots, 193, \omega_{0, i}=2 \pi f_{0, i}\right)$.

\begin{tabular}{c|ccccccccc}
\hline fuel assembly fixed & & $f_{0, i}$ & $\delta_{i}$ & $m_{i}$ & $k_{\mathrm{f}}$ & $k_{\mathrm{s}}$ & $k_{\mathrm{r}}$ & $\sigma\left(F_{i, \text { ext }}\right)$ & $\delta t\left(F_{i, \text { ext }}\right)$ \\
& unit & $\mathrm{Hz}$ & $\%$ & $\mathrm{~kg}$ & $\mathrm{~N} / \mathrm{m}$ & $\mathrm{N} / \mathrm{m}$ & $\mathrm{N} / \mathrm{m}$ & $\mathrm{N}$ & $\mathrm{s}$ \\
\hline no & & 1.0 & 10.0 & 800 & $10^{8}$ & $10^{6}$ & $10^{8}$ & 375 & 0.1 \\
yes & & 1.0 & 10.0 & 800 & 0 & 0 & 0 & 0 & -
\end{tabular}

\subsection{Results}

Figure 3 presents the simulated fluctuations of the nodal power densities at selected locations in spectral representation with the functions APSD, $\mathrm{COH}, \mathrm{PHA}$, and $\sigma$ having their usual meaning (cf. Ref. [5]). The curves are based on a time-dependent $D Y N 3 D$ run of $100 \mathrm{~s}$ length with a thermalhydraulics time-step of $0.01 \mathrm{~s}$. The time series are cut into sweeps of 1024 values with an overlap of 512 values using a Hamming window.

All auto power spectral densities (APSDs) have a similar shape with a peak at $1 \mathrm{~Hz}$ as the only dominant detail. Above $1 \mathrm{~Hz}$, the values decrease by about 6 orders of magnitude. Above $7 \mathrm{~Hz}$, the values further decrease with a more moderate slope. The standard deviation $\sigma$ has values in the range of percents and its maximum values at mid height (between level 14 and 25, cf. Fig. 1b) in the outer core regions. From the more central positions to the outer core regions, the standard deviation varies by factor $\approx 3.5$.

Correlating the time-series yields very high coherence $(\mathrm{COH})$ values $(\lesssim 1)$ for all pairings at all frequencies. Correlating inner and outer locations, the coherence is slightly lower $(\approx 0.7)$. The phase (PHA) is either $\pi$ for pairings in opposite core halves or 0 for pairings within one core half.

\subsection{Discussion}

The simulation of the "fuel-assembly-pitch effect" reproduces main details of the described phenomenology of the neutron flux fluctuations measured in KWU PWRs, including the range and the radial dependence of the fluctuation magnitudes. Therefore, the synchronized vibration of great parts of the fuel-assembly ensemble can be considered a promising approach for being the cause of the unexplained long-range neutron flux fluctuation correlations. Furthermore, a variation of the fuel-assembly parameters, e. g. corresponding to a new fuel-assembly type, have an impact on the fuel-assembly vibration amplitudes and thus on the neutron flux fluctuation magnitude. 


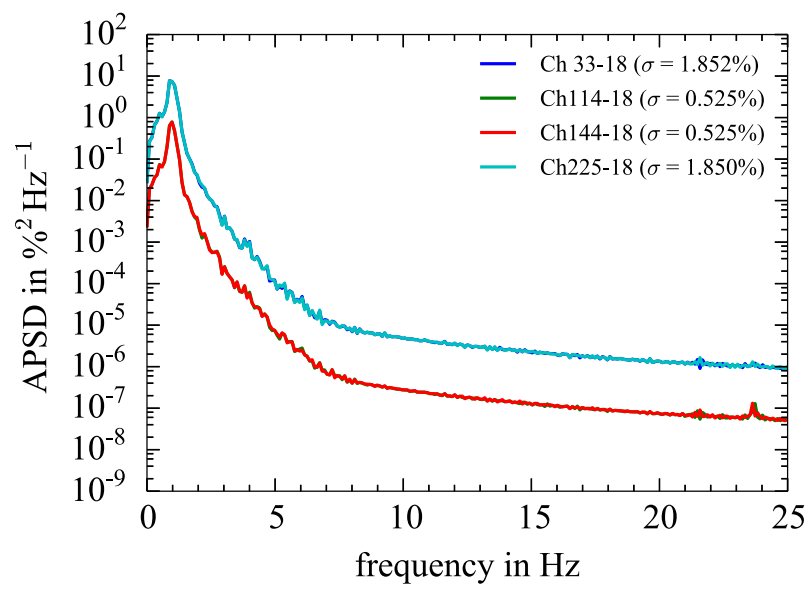

(a) APSD in radial comparison.

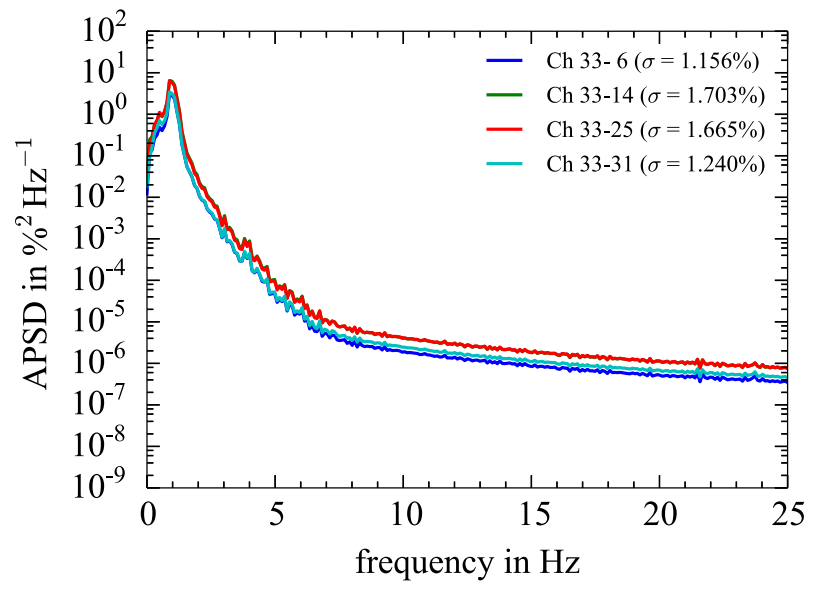

(c) APSD in axial comparison.

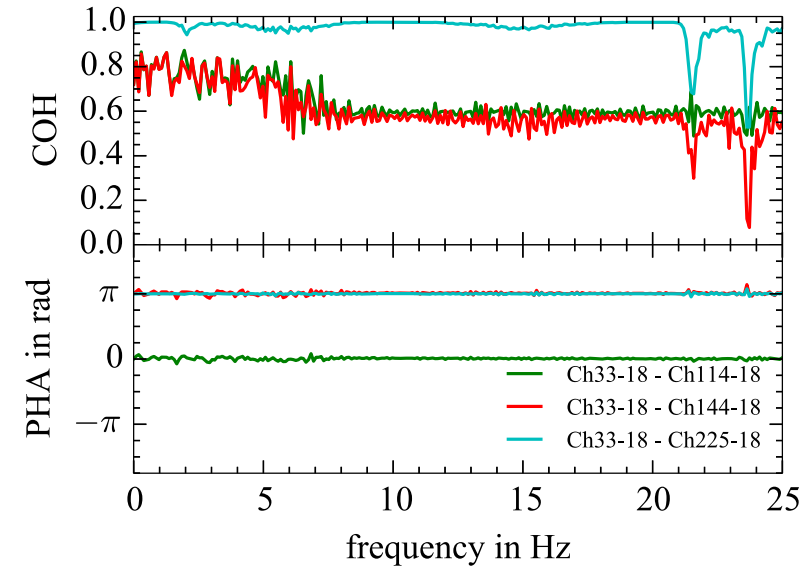

(b) Coherence and phase in radial comparison.

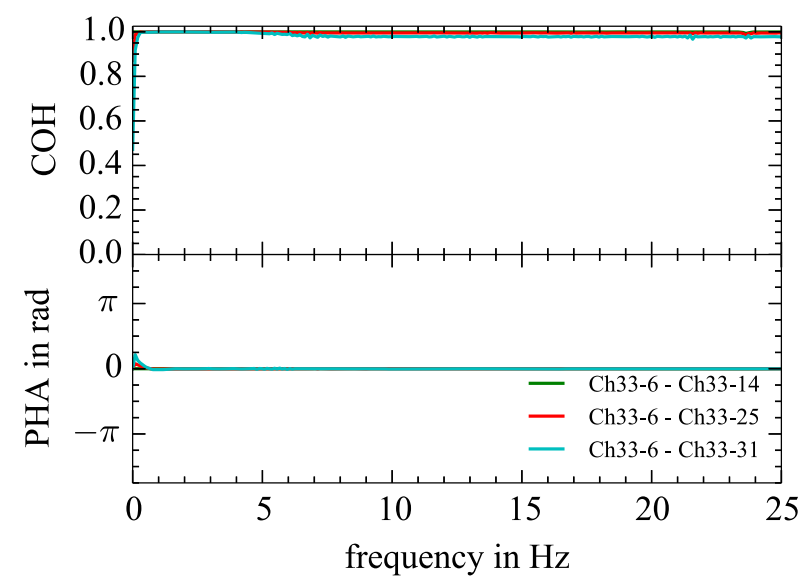

(d) Coherence and phase in axial comparison.

Figure 3: Nodal power-density fluctuations simulated with $D Y N 3 D$ assuming a synchronized fuel-assembly vibration under fixation of the outermost fuel-assembly ring.

The peak at $1 \mathrm{~Hz}$ corresponds to the chosen natural frequency $f_{0, i}$ of the fuel assemblies. Measurements at a 4-loop Vorkonvoi reactor give a similar peak at $0.8 \mathrm{~Hz}$ (cf. e. g. Ref. [5]), which has sometimes been related to fuel-assembly vibration as well (cf. e.g. Ref. [11,12]). From the authors' point of view, the proposed relation should be further investigated, e. g. by dedicated measurements at multiple reactor states varying in coolant-mass flow (3-loop vs. 4-loop operation) and reactor power (100\% vs. less than $100 \%)$.

The simulation shown in this paper aims on testing the "fuel-assembly-pitch effect" in general. It is understood that the mechanical model, its coupling to the neutron kinetics, and the drive by the coolant flow need to be improved to provide quantitative results.

\section{CONCLUSIONS}

Neutron flux fluctuations in KWU PWRs exhibit correlation patterns and magnitude changes that are not entirely understood. The hypothesis of a synchronized vibration of all fuel assemblies but the outermost ring triggering these phenomena was tested via $D Y N 3 D$, confirming the relevance of this scenario. Improvements of the modelling are required for quantitative studies. For proving the hypothesis, dedicated measurements in the plant are proposed. 


\section{ACKNOWLEDGEMENTS}

This work was supported by the German Federal Ministry for Economic Affairs and Energy (Project NEUS, grant number 1501587). The responsibility for the content of this publication lies with the authors.

\section{REFERENCES}

[1] (German) Reactor Safety Commission (RSK). "PWR neutron flux oscillations." RSK Statement (457th meeting on 11042013) (2013).

[2] G. Verdú, M. García-Fenoll, A. Abarca, R. Miró, and T. Barrachina. "Study of the noise propagation in PWR with coupled codes." In International Conference on Mathematics and Computational Methods Applied to Nuclear Science and Design (M \& C 2011). Rio de Janeiro, Brasil, Mayo 8-12 2011. American Nuclear Society (ANS) (2011).

[3] M. Seidl, K. Kosowski, U. Schüler, and L. Belblidia. "Review of the historic neutron noise behavior in German KWU built PWRs." Progress in Nuclear Energy, volume 85, pp. 668 675 (2015).

[4] J. Bermejo, C. Montalvo, and A. Ortego. "On the possible effects contributing to neutron noise variations in KWU-PWR reactor: Modelling with S3K." Progress in Nuclear Energy, volume 95, pp. 1 - 7 (2017).

[5] M. Viebach, C. Lange, N. Bernt, M. Seidl, D. Hennig, and A. Hurtado. "Simulation of low-frequency PWR neutron flux fluctuations." Progress in Nuclear Energy, volume 117, p. 103039 (2019).

[6] D. Chionis, A. Dokhane, H. Ferroukhi, G. Girardin, and A. Pautz. "PWR NEUTRON NOISE PHENOMENOLOGY: PART II QUALITATIVE COMPARISON AGAINST PLANT DATA." In PHYSOR 2018: Reactor Physics paving the way towards more efficient systems, Cancun, Mexico, April 22-26, 2018 (2018).

[7] U. Rohde, S. Kliem, U. Grundmann, S. Baier, Y. Bilodid, S. Duerigen, E. Fridman, A. Gommlich, A. Grahn, L. Holt, Y. Kozmenkov, and S. Mittag. "The reactor dynamics code DYN3D models, validation and applications." Progress in Nuclear Energy, volume 89, pp. 170 - 190 (2016).

[8] A. Wanninger, M. Seidl, and R. Macián-Juan. "Mechanical analysis of the bow deformation of a row of fuel assemblies in a PWR core." Nuclear Engineering and Technology, volume 50(2), pp. $297-305$ (2018). Special issue on the water reactor fuel performance meeting 2017 (WRFPM 2017).

[9] Studsvik Scandpower, Inc. "CASMO5. A FUEL ASSEMBLY BURNUP PROGRAM."

[10] The MathWorks Inc., Natick. MATLAB R2016b (2016).

[11] J. Runkel. Rauschanalyse in Druckwasserreaktoren. Ph.D. thesis, Universitt Hannover (1987).

[12] D. Stegemann and J. Runkel. "Experience with Vibration Monitoring in German PWRs Obrigheim, Grohnde, Brokdorf and Emsland." In SMORN VII. A SYMPOSIUM ON NUCLEAR REACTOR SURVEILLANCE AND DIAGNOSTICS, Avignon, France, 19-23 June 1995, volume 1, pp. 23-31 (1996). 